\title{
CONTRIBUTO ALLA CONOSCENZA DEI BOSCHI A QUERCUS ILEX DELLA SICILIA
}

\author{
Giuseppina BARTOLO, Salvatore BRULLO, Pietro MINISSALE \& \\ Giovanni SPAMPINATO
}

\begin{abstract}
RIASSUNTO: In questo lavoro vengono esaminati i boschi a Quercus ilex presenti in Sicilia. Oltre alle associazioni in precedenza già osservate per questo territorio, viene segnalata per la prima volta la presenza dell'Ostrio-Quercetum ilicis e inoltre sono descritte alcune nuove subassociazioni relative al Querco-Teucrietum siculi e all'Aceri obtusati-Ostryetum carpinifoliae. Ciascuna associazione viene esaminata sotto il profilo syntassonomico, ecologico e sindinamico.
\end{abstract}

Parole chiave: Sicilia, fitosociologia, boschi Quercus ilex.

SUMMARY: In this paper the Quercus ilex woods occurring in Sicily were examined. Apart from the associations previosly observed in this territory, the presence of Ostryo-Quercetum ilicis is reported for the first time; besides some new subassociations of the Querco-Teucrietum siculi and of the Aceri obtusati-Ostryetum carpinifoliae are described. Syntaxonomic, ecological and syndynamic considerations are given for every associations.

Key words: Sicily, Phytosociology, Quercus ilex woods.

\section{N T R O D U Z I O N E}

I boschi a Quercus ilex, che rappresentano uno degli aspetti forestali più tipici e peculiari fra quelli presenti nel bacino del Mediterraneo, in Sicilia risultano attualmente poco diffusi e in genere abbastanza localizzati (Fig. 1). In molti casi si tratta di formazioni relitte, le quali si sono potute conservare in quanto localizzate per lo più in aree impervie e rocciose e pertanto poco interessate da processi di antropizzazione, o in stazioni montane quindi non idonee climaticamente allo sfruttamento agricolo.

Sotto il profilo fitosociologico questo tipo di vegetazione boschiva è stato oggetto, per quanto riguarda il territorio siculo, di numerosi contributi che hanno portato alla identificazione e descrizione di varie associazioni (cfr. Furnari 1965, Gentile 1969, Poli \& Maugeri 1974, Brullo \& Ronsisvalle 1975, Brullo et al. 1977, Barbagallo et al. 1979, Poli et al. 1979, Brullo \& Marcenò 1983 e 1985, 
Brullo 1984). Pertanto, sulla base delle attuali conoscenze e di numerosi dati inediti, si ritiene utile presentare uno studio riguardante soprattutto l'aspetto sintassonomico, sindinamico ed ecologico delle leccete presenti in Sicilia e isole vicine.

In particolare vengono esaminate sia le formazioni pure a Quercus ilex sia quelle in cui questa specie rispetto ad altre essenze arboree ha un ruolo dominante o comunque è abbastanza rappresentata come numero di individui. Inoltre per il rilevante ruolo che le leccete assumono in seno al paesaggio naturale del territorio, significativa è la posizione dinamica che le singole associazioni ricoprono in relazione alle serie evolutive nelle quali si inseriscono.

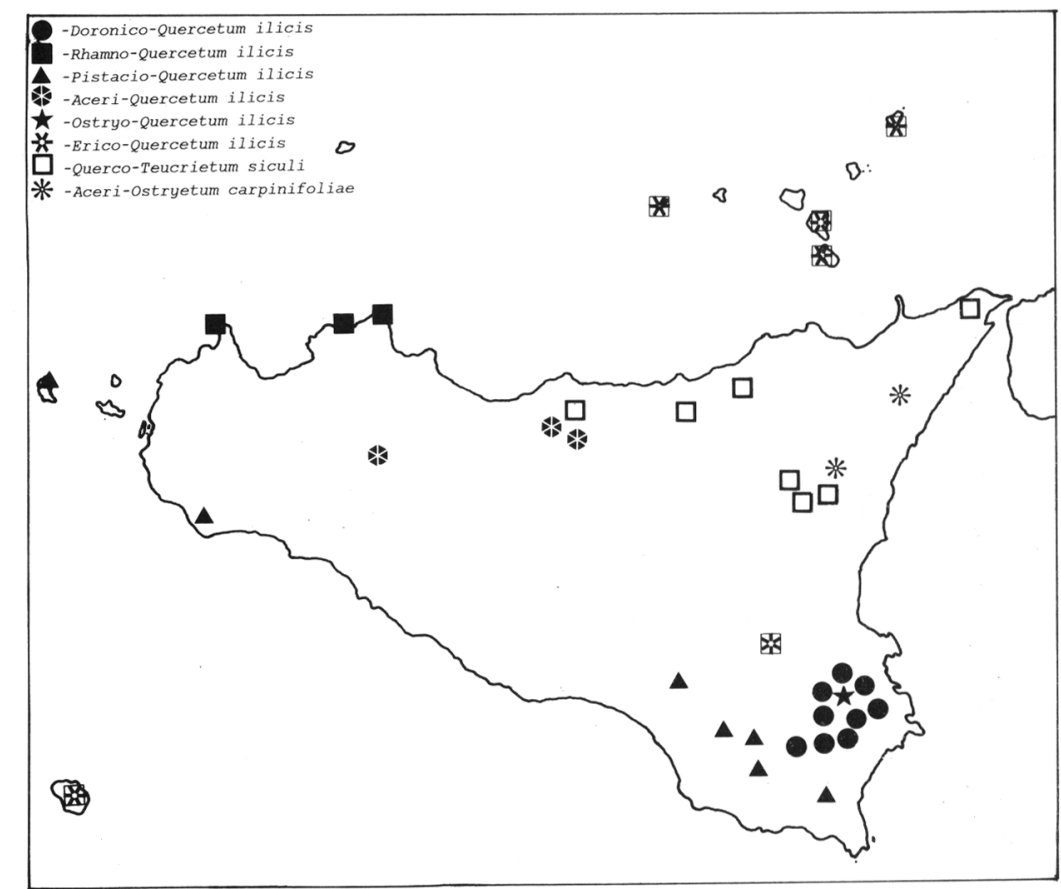

Figura 1.- Distribuzione in Sicilia delle associazioni a Quercus ilex.

Nell'ambito dei boschi a Quercus ilex presenti in Sicilia, formazioni appartenenti tutte ai Quercetea ilicis, possono essere distinti aspetti nettamente acidofili, legati a substrati silicei o a suoli lisciviati, i quali sono stati inclusi nell'EricoQuercion ilicis, e aspetti basifili tipici dei substrati calcarei o comunque dei suoli basici o neutri, rientranti questi nel Quercion ilicis (cfr. Brullo \& Marcenò 1985). Queste alleanze nel complesso si differenziano abbastanza bene tra di loro sia sotto il profilo eco-pedologico che floristicamente per la presenza, soprattutto negli aspetti dell'Erico-Quercion ilicis, di un ricco contingente di specie calcifughe in genere assenti o rare nelle formazioni calcicole.

Le associazioni oggetto di questa indagine sono riportate nel seguente quadro sintassonomico: 
Numero del rilievo

Quota (m)

Superficie (mq)

Copertura (\%)

Inclinazione $\left({ }^{\circ}\right)$

Esposizione $\begin{array}{llllllllllll}1 & 2 & 3 & 4 & 5 & 6 & 7 & 8 & 9 & 10 & 11 & 12\end{array}$

$\begin{array}{llllllllllll}380 & 670 & 680 & 500 & 560 & 570 & 590 & 560 & 550 & 630 & 620 & 500\end{array}$

200100100100200200200150100100100100 $10010080 \quad 10080 \quad 90 \quad 90 \quad 100100100100100$ $\begin{array}{lllllllllllll}25 & 20 & 20 & 20 & 20 & 25 & 20 & 20 & 15 & -\end{array}$

Car. Associazione

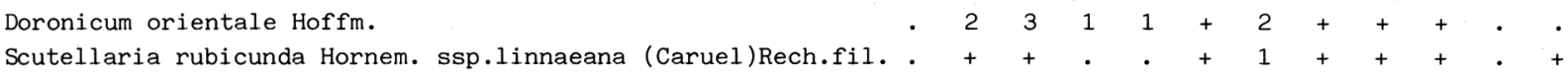
Aristolochia clusii Lojacono

Car. All. (QUERCION ILICIS)

e Ord.. (QUERCETALIA ILICIS)

Quercus ilex L.

Tamus communis L.

Cyclamen repandum Sibth. \& Sm.

Ruscus aculeatus L.

Hedera helix L.

Carex distachya Desf.

Asplenium onopteris L.

Cyclamen hederifolium Aiton

Viola dehnhardtii Ten.

Carex hallerana Asso

Quercus ilex L. (pl)

Car. Cl. (QUERCETEA ILICIS)

Quercus virgiliana Ten..

Rubia peregrina L.

Asparagus acutifolius L.

Euphorbia characias L.

Smilax aspera L.

Pistacia terebinthus $\mathrm{L}$.

Phillyrea angustifolia L.

Rhamnus alaternus L.

Pimpinella peregrina $\mathrm{L}$.

Pistacia lentiscus L.

Osyris alba L.

Teucrium flavum L.

Arisarum vulgare Targ.-Tozz.

Daphne gnidium L.

Lonicera implexa Aiton

Lonicera etrusca Santi

Phillyrea latifolia L.

Altre specie

Crataegus monogyna Jacq.

Ranunculus neapolitanus Ten.

Silena italica (L.) Pers.

Allium subhirsutum L.

Dryopteris pallida Bory

Rosa sempervirens L.

Brachypodium sylvaticum (Hudson) Beauv.

Geranium robertianum L.

Rubus ulmifolius Schott

Sanguisorba minor Scop.

Melica minuta L.

Galium corrudifolium Vill.

Acanthus mollis L.

Cistus salvifolius L.

Crepis leontodoides All.

Geranium molle L.

Selaginella denticulata (L.) Link

Cistus incanus $\mathrm{L}$.

Ceterach officinarum DC.

Torilis arvensis (Huds.) Link

Clematis vitalba $\mathrm{L}$.

Hypochoeris laevigata (L.)Ces.Pass.\& Gib.

Sporadiche

$\begin{array}{llllllllllll}4 & 5 & 4 & 5 & 4 & 4 & 4 & 5 & 5 & 5 & 5 & 5 \\ 1 & + & + & 2 & 1 & + & 1 & 1 & 2 & 2 & 1 & 2 \\ 2 & + & + & + & + & + & + & + & + & 1 & 1 & + \\ 2 & 1 & + & 1 & 1 & + & + & + & . & 1 & 1 & 1 \\ 1 & 1 & + & 2 & + & + & . & . & . & + & 2 & 1 \\ . & + & 1 & + & + & + & + & + & . & + & + & \cdot \\ 1 & . & + & + & 1 & + & + & . & 1 & . & . & \cdot \\ \cdot & 1 & + & 1 & . & . & . & + & . & . & . & . \\ + & . & . & . & . & . & . & . & . & 1 & + & + \\ . & + & . & . & . & . & . & . & + & + & . & . \\ 1 & . & . & . & . & . & . & . & . & . & . & .\end{array}$

Ril. 1-12 Provincia di Siracusa (Barbagallo, Brullo \& Fagotto 1979) 
Tab. 2 - OSTRYO-QUERCETUM ILICIS

Numero del rilievo

$\begin{array}{lllll}1 & 2 & 3 & 4 & 5\end{array}$

Quota (m)

$\begin{array}{lllll}410 & 420 & 450 & 450 & 420\end{array}$

Superficie (mq)

100100100100100

Copertura (\%)

Inclinazione $\left({ }^{\circ}\right)$

$10010095 \quad 100100$

Esposizione

$\begin{array}{lllll}30 & 40 & 30 & 40 & 30\end{array}$

$\begin{array}{lllll}\mathrm{N} & \mathrm{N} & \mathrm{N} & \mathrm{N} & \mathrm{NE}\end{array}$

Car. Associazione

Ostrya carpinifolia Scop.

$\begin{array}{lllll}2 & 1 & 3 & 2 & 1\end{array}$

Car. All. (QUERCION ILICIS),

Ord. (QUERCETALIA ILICIS) e

Cl. (QUERCETEA ILICIS)

Quercus ilex L.

Smilax aspera L.

Fraxinus ornus L.

Asparagus acutifolius L.

Rubia peregrina L.

Ruscus aculeatus L.

Rhamnus alaternus L.

Coronilla emerus L.

Bupleurum fruticosum L.

Asplenium onopteris L.

Pistacia lentiscus L.

Rosa sempervirens L.

Teucrium flavum L.

Tamus communis L.

Osyris alba L.

Pistacia terebinthus L.

Phillyrea latifolia L.

Euphorbia characias L.

Viola dehnhardtii Ten.

Carex distachya Desf.

Clematis cirrhosa L.

Arisarum vulgare Targ. Tozz.

Altre specie

Brachypodium sylvaticum (Hudson) Beauv. $22+++$

Hedera helix L.

Aristolochia sempervirens L.

Acanthus molis L.

Dryopteris pallida Bory

Sporadiche

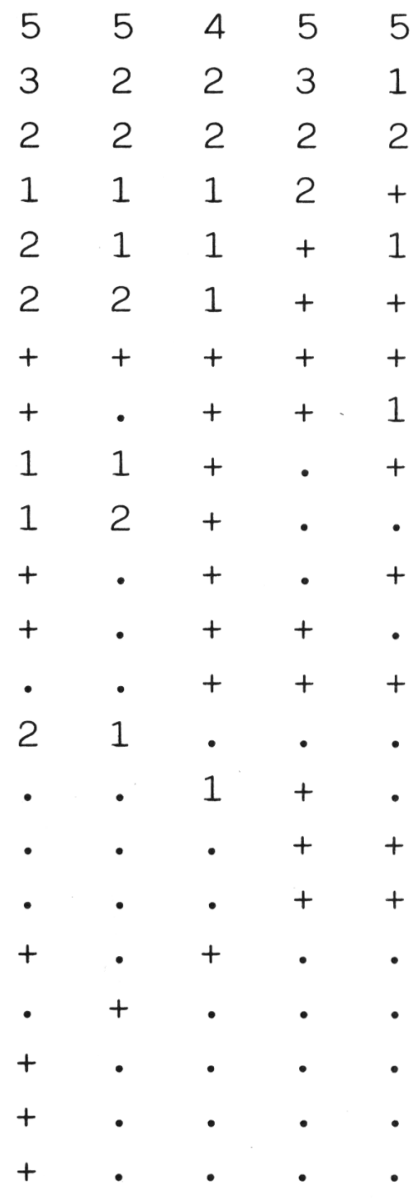

Ril. 1-6 Valle dell'Anapo, Siracusa 


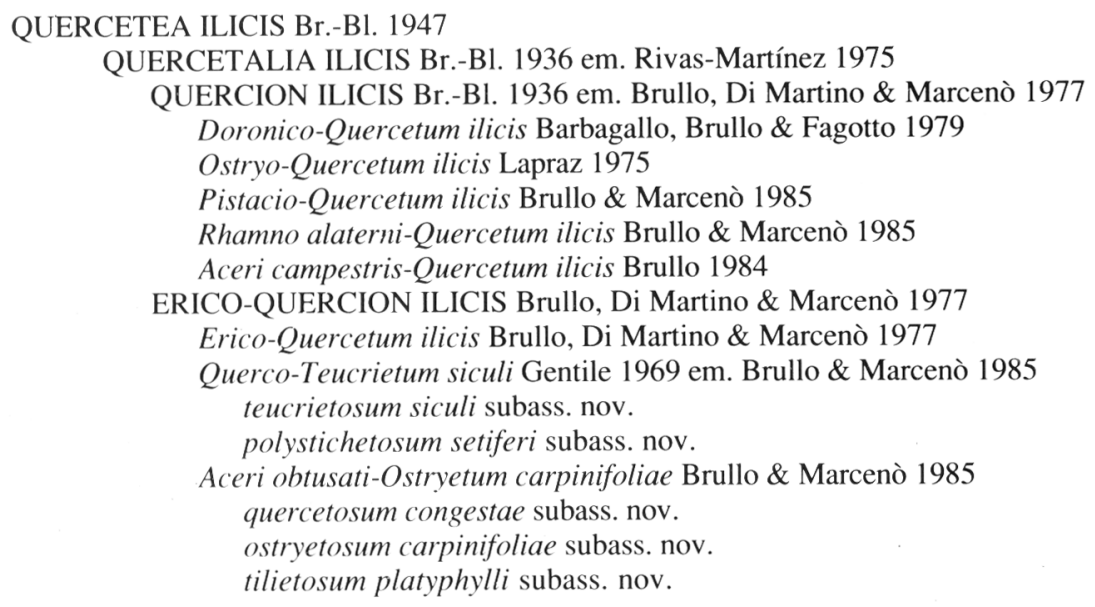

A - Doronico-Quercetum ilicis Barbagallo, Brullo \& Fagotto 1979 (Tab. 1, Fig. 2)

Si tratta di una lecceta con caratteri mesofili, la quale risulta localizzata su calcari miocenici, limitatamente a stazioni fresche ed ombreggiate come quelle poste sul fondo e sulle pendici settentrionali dei valloni. Essa è stata rilevata da Barbagallo et al. (1979) nel territorio ibleo (Sicilia sud-orientale), dove è circoscritta alle profonde valli fluviali (localmente chiamate cave) del versante ionico, area questa interessata da precipitazioni medie annue comprese fra 700 e $1000 \mathrm{~mm}$.

Floristicamente l'associazione risulta differenziata dalla presenza di Doronicum orientale, specie distribuita nel Mediterraneo nord-orientale e dalle endemiche Scutellaria rubicunda ssp. linneana e Aristolochia clusii.

Il Doronico-Quercetum ilicis in condizioni di elevata umidità edafica, come lungo le sponde dei fiumi, prende contatto con il Platano-Salicetum pedicellatae Barbagallo, Brullo \& Fagotto 1979, ripisilva del Platanion orientale I. \& V. Karpati 1961 (cfr. Barbagallo et al. 1979a). Nelle stazioni più soleggiate dei valloni, come lungo le pendici meridionali, la lecceta viene normalmente sostituita dall'OleoQuercetum virgilianae Brullo 1984, vegetazione boschiva decidua di tipo climacico marcatamente termofila. Negli ambienti più rocciosi dei valloni si insedia invece l'Oleo-Euphorbietum dendroidis Trinajstic 1974 vegetazione arbustiva xerofila di tipo edafico dell'Oleo-Ceratonion, la quale viene sostituita sulle pareti verticali dal Putorio-Micromerietum microphyllae Brullo \& Marcenò 1979, associazione casmofila degli Asplenietalia glandulosi Br.-Bl. 1934. La distruzione della lecceta favorisce l'insediamento di una macchia molto peculiare, rappresentata dal Salvio-Phlomidetum fruticosae Barbagallo, Brullo \& Fagotto 1979, alla quale successivamente si sostituisce, con il perdurare dei processi di degradazione, la prateria ad Ampelodesmos mauritanicus. 


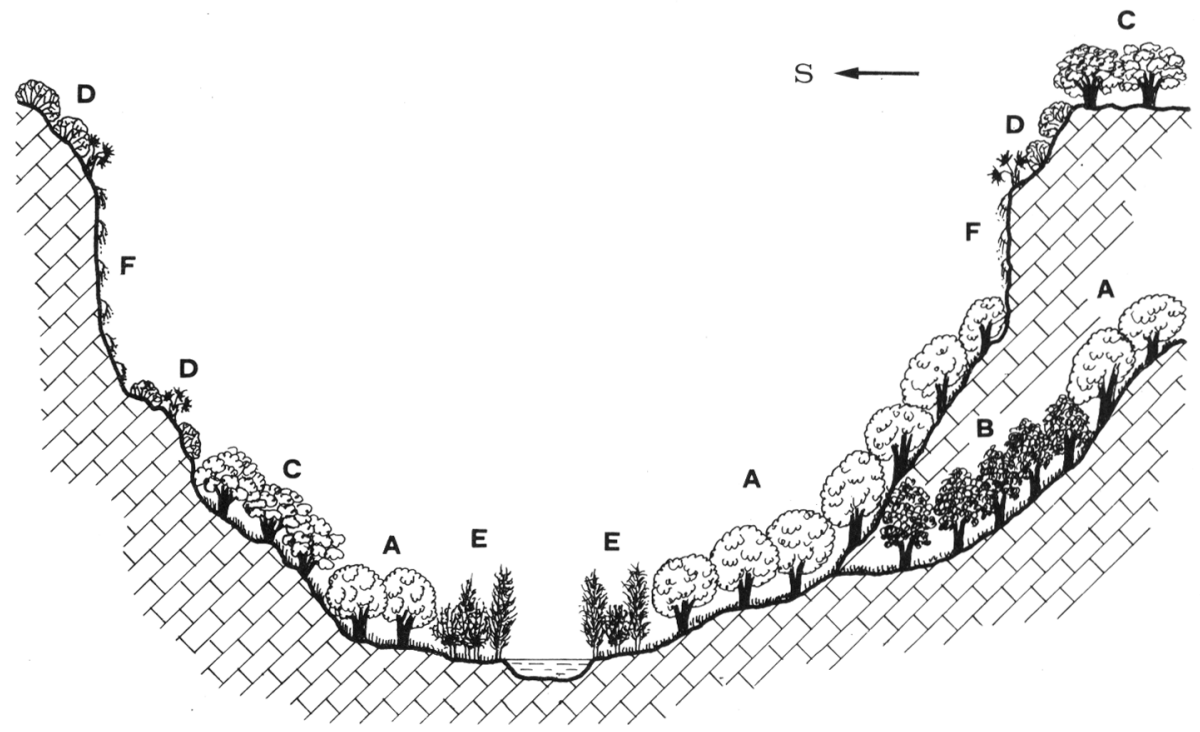

Figura 2.- Transect della vegetazione potenziale delle cave iblee (Sicilia sud-orientale): A. Doronico-Quercetum ilicis; B. Ostryo-Quercetum ilicis; C. Oleo-Quercetum virgilianae; D. Oleo-Euphorbietum dendroidis; E. Platano-Salicetum pedicellatae; F. PutorioMicromerietum microphyllae.

\section{B - Ostryo-Quercetum ilicis Lapraz 1975 (Tab. 2, Fig. 2)}

Questa associazione, in precedenza nota solo per la Provenza (Lapraz 1975), la Dalmazia (Trinajstic 1977) e l'Appennino centro-meridionale (Blasi et al. 1981, Biondi 1982, Ballelli \& Biondi 1982, Signorello 1985), viene segnalata per la prima volta in Sicilia. Si tratta di una formazione mista a leccio e ostria di tipo basifilo, la quale nel territorio è stata rinvenuta nell'area iblea limitatamente ad alcune cave del siracusano.

L'Ostryo-Quercetum ilicis si localizza sui versanti settentrionali in corrispondenza dei tratti più ombreggiati e riparati, come le aree di compluvio dove si registra anche una maggiore umitidà edafica. Questa associazione, che si differenzia dalle altre leccete calcicole per la presenza di Ostrya carpinifolia, assume pertanto un significato prettamente edafico. Trattandosi di una formazione marcatamente mesofila, essa nel territorio sostituisce il Doronico-Quercetum ilicis in situazioni di una migliore disponibilità idrica del suolo. La degradazione di questa vegetazione favorisce l'insediamento di aspetti del Pruno-Rubion ulmifolii O. Bolòs 1954, che costituisce dei densi ed intricati arbusteti lianosi osservabili sia ai margini che nelle schiarite delle aree boschive. 
Numero del rilievo

Quota (m)

Superficie (mq)

Copertura (\%)

Diff. Associazione

Pistacia lentiscus L.

Car. All. (QUERCION ILICIS)

e Cl. (QUERCETEA ILICIS)

Quercus ilex L.

Asparagus acutifolius L.

Cyclamen repandum Sibth. \& Sm.

Arisarum vulgare Targ.-Tozz.

Rubia peregrina L.

Calicotome infesta (C. Presl) Guss.

Phillyrea latifolia L.

Tamus communis L.

Phillyrea angustifolia L.

Rhamnus alaternus L.

Ruscus aculeatus L.

Osyris alba L.

Lonicera implexa Aiton

Smilax aspera L.

Daphne gnidium L.

Euphorbia characias L.

Carex distachya Desf.

Daphne sericea Vahl

Carex hallerana Asso

Ruta chalepensis L.

Asplenium onopteris L.

Rosa sempervirens L.

\section{Trasgr. PISTACIO-RHAMNETALIA ALATERNI}

Teucrium fruticans L.

Prasium majus L.

Euphorbia dendroides L.

Olea europaea L. var. sylvestris Brot.

Ephedra fragilis Desf.

Chamaerops humilis L.

Quercus calliprinos Webb

Ceratonia siliqua L.

Clematis cirrhosa L.

\section{Altre specie}

Allium subhirsutum L.

Ampelodesmos mauritanicus (Poiret)Dur.\& Sch.

Pinus halepensis Miller

Carex serrulata Biv.

Erica multiflora L.

Selaginella denticulata (L.) Link

Acanthus mollis $\mathrm{L}$.

Urginea maritima (L.) Baker

Cistus incanus $\mathrm{L}$.

Micromeria graeca (L.) Bentham

Rubus ulmifolius Schott

Geranium purpureum Vill.

Polypodium australe Fée

Crataegus monogyna Jacq.

Cistus salvifolius L.

Sporadiche

Ril. 1-3 S.Pietro, Caltagirone (FURNARI 1965)

Ril. 4-5 Gorghi Tondi, Mazara del Vallo (BRULLO \& RONSISVALLE 1975)

Ril. 6-7 Marettimo (BRULLO \& MARCENO'1983)

Ril. 8 Modica (BRULLO \& MARCENO' 1985)

Ril. 9-14 Vittoria 
Tab. 4 - RHAMNO-QUERCETUM ILICIS

Numero del rilievo

Quota (m)

Superficie (mq)

Copertura (\%)

Diff. Associazione

Viburnum tinus. L.

Rhamnus alaternus L.

Car. All. (QUERCION ILICIS)

e Cl. (QUERCETEA ILICIS)

Quercus ilex L.

Fraxinus ornus L.

Asparagus acutifolius L.

Rubia peregrina L.

Smilax aspera L.

Arisarum vulgare Targ.-Tozz.

Tamus communis L.

Ruta chalepensis L.

Rosa sempervirens L.

Cyclamen repandum Sibth. et Sm.

Carex distachya Desf.

Lonicera implexa Aiton

Laurus nobilis L.

Clematis cirrhosa L.

Ruscus aculeatus L.

Daphne gnidium L.

Asplenium onopteris L.

Lonicera etrusca Santi

Phillyrea latifolia L.

Pistacia terebinthus L.

Euphorbia characias $L$.

Trasgr. PISTACIO-RHAMNETALIA ALATERNI

Teucrium flavum L.

Euphorbia dendroides L.

Anagyris foetida L.

Prasium majus L.

Ceratonia siliqua $\mathrm{L}$.

Olea europaea L. var. sylvestris Brot.

Pistacia lentiscus L.

Altre specie

Inula conyza DC.

Allium subhirsutum L.

Acanthus mollis L.

Geranium purpureum Vill.

Rubus ulmifolius Schott

Anemone hortensis L.

Rhus coriaria L.

Urginea maritima (L.) Baker

Blackstonia perfoliata (L.) Hudson

Ampelodesmos mauritanicus (Poiret)Dur.\& Sch.

Aetheorrhiza bulbosa (L.) Cass.

Calamintha nepeta (L.) Savi

Hypericum perforatum L.

Crataegus monogyna Jacq.

Polypodium cambricum L.

Sporadiche $\begin{array}{llllllllll}1 & 2 & 3 & 4 & 5 & 6 & 7 & 8 & 9 & 10\end{array}$

$\begin{array}{llllllllll}110 & 100 & 90 & 100 & 120 & 80 & 100 & 170 & 150 & 120\end{array}$

250250250200250250200100150100

$\begin{array}{llllllllll}60 & 40 & 70 & 70 & 60 & 40 & 60 & 40 & 60 & 60\end{array}$

$\begin{array}{llllllllll}2 & 2 & 3 & 3 & 1 & 3 & 3 & 2 & + & + \\ 1 & 1 & 2 & 2 & 2 & 2 & 1 & 2 & 1 & 2\end{array}$

Ril. 1-10 M. Pellegrino, Palermo (BRULLO \& MARCENO' 1985) 


\section{C-Pistacio-Quercetum ilicis Brullo \& Marcenò 1985 (Tab. 3, Fig. 3 e 4)}

Nella Sicilia occidentale e meridionale, più precisamente nel tratto compreso fra Trapani e Capo Passero, le leccete sono nel complesso abbastanza rare e in genere, quando sono presenti si localizzano sul fondo dei valloni e sulle pendici settentrionali più o meno ombreggiate dei rilievi. Si tratta di formazioni marcatamente termofile rientranti nel Pistacio-Quercetum ilicis, associazione calcicola legata a substrati calcarei, calcarenitici e marnosi. Essa si differenzia dalle altre leccete basifile per la presenza di Pistacia lentiscus e di un ricco contingente di sclerofille dei Pistacio-Rhamnetalia alaterni Rivas-Martínez 1975. Dal punto di vista climatico essa risulta distribuita nella parte più arida del territorio siculo, la quale è caratterizzata da precipitazioni medie annue di $400-500 \mathrm{~mm}$.

Come la maggior parte delle leccete della Sicilia, anche questa associazione ha un significato azonale. Essa si rinviene infatti all'interno dell'area climacica dell'Oleo-Quercetum virgilianae, per quanto riguarda almeno le stazioni più interne. Esempi di questa lecceta sono stati osservati pure in tratti più costieri interessati da formazioni xerofile dei Pistacio-Rhamnetalia alaterni. In particolare per quanto riguarda la Sicilia meridionale, il Pistacio-Quercetum ilicis si rinviene in seno al Thymo-Pinetum halepensis De Marco e Caneva 1985, mentre nella Sicilia occidentale è localizzato all'interno del Chamaeropo-Quercetum calliprini Brullo \& Marcenò 1985. La degradazione del Pistacio-Quercetum ilicis favorisce l'insediamento di garighe termo-xerofile attribuibili per lo più al Rosmarino-Thymetum capitati Furnari 1965.

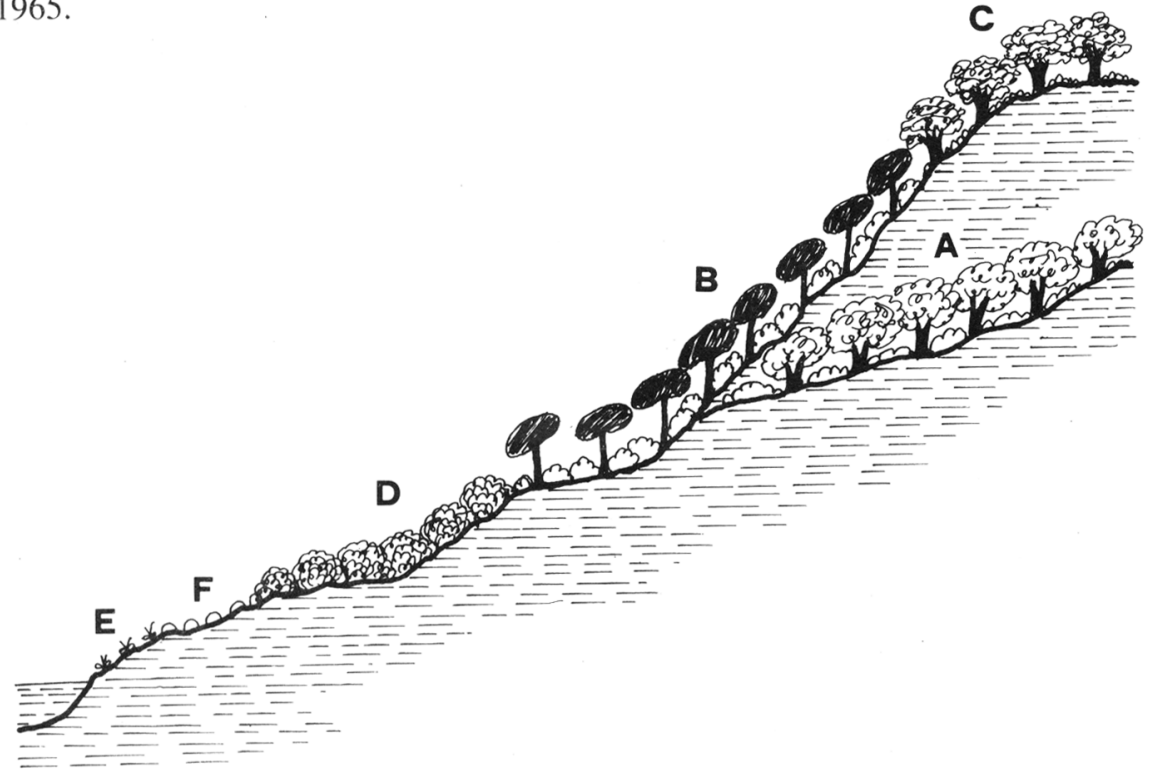

Figura 3.- Transect della vegetazione potenziale della fascia costiera della Sicilia meridionale: A. Pistacio-Quercetum ilicis; B. Thymo-Pinetum halepensis; C. Oleo-Quercetum virgilianae; D. Myrto-Lentiscetum; E. Limonietum hyblaei; F. Thymelaeo-Helichrysetum siculi. 


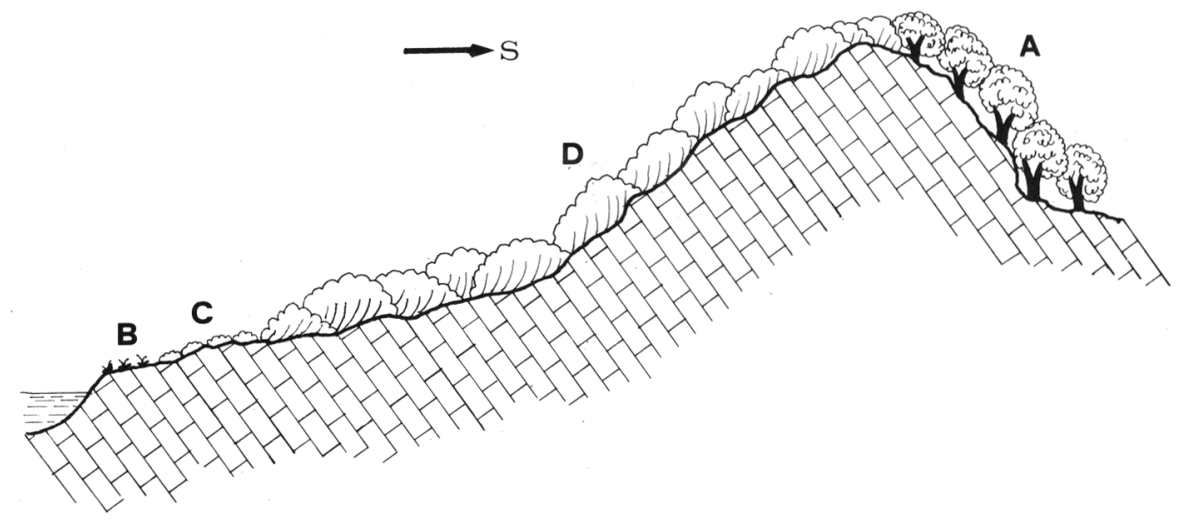

Figura 4.- Transect della vegetazione potenziale della fascia costiera della Sicilia occidentale: A. Pistacio-Quercetum ilicis; B. Limonietum mazarae; C. Thymelaeo-Helichrysetum siculi; D. Chamaeropo-Quercetum calliprini.

D - Rhamno alaterni-Quercetum ilicis Brullo \& Marcenò 1985 (Tab. 4, Fig. 5)

Questa associazione è stata rilevata sui versanti settentrionali dei monti costieri della Sicilia nord-occidentale. Essa si localizza su substrati calcarei o dolimitici in corrispondenza dei brecciai che ricoprono le pendici dei rilievi. Si tratta di materiale clastico proveniente dall'erosione e disfacimento delle sovrastanti pareti rocciose. Di un certo rilievo è in questa associazione la presenza di Viburnum tinus, specie rara in Sicilia la quale si rinviene con una certa frequenza solo nelle stazioni in oggetto. Abbastanza diffusi e talora dominanti risultano pure Rhamnus alaternus e Fraxinus ornus, che assieme a Quercus ilex caratterizzano fisionomicamente questa formazione.

Il Rhamno-Quercetum ilicis viene normalmente sostitutito in situazioni di cengia e in ambienti semirupestri dall'Oleo-Euphorbietum dendroidis, associazione essenzialmente edafica la quale a sua volta è rimpiazzata sulle pareti rocciose verticali dallo Scabioso-Centauretum ucriae Brullo \& Marcenò 1979, vegetazione rupicola degli Asplenietalia glandulosi. Nelle stazioni più prossime alla riva, la lecceta prende contatto con il Pistacio-Chamaeropetum humilis Brullo \& Barcenò 1985, macchia xerofila essenzialmente costiera.

Questa lecceta a causa dell'azione di disturbo antropico, come tagli e incendi, è ormai diventata piuttosto rara e al suo posto si rinvengono estese praterie ad Ampelodesmos mauritanicus, vegetazione erbacea di tipo perenne la cui diffusione, come pure il suo mantenimento nel tempo, è favorita dai periodici incendi. 
Tab. 5 - ACERI CAMPESTRIS-QUERCETUM ILICIS

Numero del rilievo

Quota (m)

Superficie (mq)

Copertura (\%)

Inclinazione $\left({ }^{\circ}\right)$

Esposizione

Car. e diff. Associazione

Acer campestre L.

Acer monspessulanum L.

Ilex aquifolium L.

Sorbus graeca (Spach) Kotscky

Car. All. (QUERCION ILICIS)

e Cl. (QUERCETEA ILICIS)

Quercus ilex L.

Ruscus aculeatus L.

Cyclamen repandum Sibth. \& Sm.

Paeonia russii Biv.

Euphorbia characias L.

Carex distachya Desf.

Thalictrum calabricum Sprengel

Viola dehnhardtii Ten.

Tamus communis L.

Rubia peregrina L.

Asplenium onopteris L.

Lonicera etrusca Santi

Coronilla emerus L.

Trasgr. QUERCO-FAGETEA

Hedera helix L.

Brachypodium sylvaticum (Hudson) Beauv.

Clematis vitalba $\mathrm{L}$.

Daphne laureola L.

Rubus canescens DC.

Lamium flexuosum $\mathrm{L}$.

Milium effusum L.

Chaerophyllum temulum L.

Geum urbanum L.

Epipactis helleborine (L.) Crantz

Altre specie

Crataegus monogyna Jacq

Prunus spinosa L.

Poa sylvicola Guss.

Rosa canina L.

Bellis perennis L.

Trifolium pratense L.

Malus sylvestris Miller

Calamintha nepeta (L.) Savi

Crataegus oxycantha L.

Stachys germanica L. var. dasyanthes (Rafin.)Briq. Orobanche sp.

Silene sicula Ucria

Origanum vulgare L.

Agrimonia eupatoria L.

Pteridium aquilinum (L.) Kuhn

Crepis leontodontoides All.

Cynosurus elegans Desf.

Daucus carota L. s.l.

Lapsana communis L.

Allium subhirsutum L.

Asplenium trichomanes L.

Asperula laevigata L.

Myosotis gussonei (Nic.) Béguinot

Sporadiche

$\begin{array}{lllllll}1 & 2 & 3 & 4 & 5 & 6 & 7 \\ 1200 & 1150 & 1100 & 1310 & 1220 & 1400 & 1300 \\ 100 & 100 & 100 & 100 & 100 & 200 & 100 \\ 100 & 100 & 100 & 100 & 100 & 80 & 100 \\ 10 & 10 & 15 & 10 & 20 & 30 & 40 \\ 0 & 0 & 0 & \text { NO } & 0 & \text { SO } & \text { N }\end{array}$

$\begin{array}{lllllll}2 & 1 & 2 & 2 & 2 & 1 & 2\end{array}$

$\begin{array}{lllllll}1 & 2 & 2 & 1 & 2 & 1 & 1\end{array}$

$\begin{array}{lllllll}1 & 1 & 2 & 3 & 2 & .\end{array}$ 
Numero del rilievo

Quota (m)

Superficie (mq)

Copertura (\%)

Diff. Associazione

Pistacia lentiscus L.

Prasium majus L.

Myrtus communis L.

Calicotome villosa (Poiret) Link

Euphorbia dendroides L.

Car. All. (ERICO-QUERCION ILICIS)

Quercus ilex L.

Arbutus unedo L.

Erica arborea L.

Melica arrecta G.Kuntze

Cytisus villosus Pourret

Teline monspessulana (L.) Koch

Car. Ord. (QUERCETALIA ILICIS)

e Cl. (QUERCETEA ILICIS)

Asparagus acutifolius L.

Daphne gnidium L.

Asplenium onopteris L.

Rubia peregrina L.

Phillyrea angustifolia L.

Smilax aspera L .

Lonicera implexa Aiton

Phillyrea latifolia L.

Arisarum vulgare Targ.-Tozz.

Carex distachya Desf.

Tamus communis L.

Asparagus officinalis L.

Fraxinus ornus L.

Rhamnus alaternus L.

Carex serrulata Biv.

\section{Altre specie}

Pteridium aquilinum (L.) Kuhn

Cistus incanus $\mathrm{L}$.

Rubus ulmifolius Schott

Cistus salvifolius L.

Pinus pinaster Aiton

Polypodium australe Fèe

Crepis leontodontoides All.

Asplenium obovatum Viv.

Geranium purpureum Vill.

Umbilicus rupestris (Salisb.) Dandy

Brachypodium sylvaticum (Hudson) Beauv.

Allium subhirsutum L.

Cistus monspeliensis L.

Geranium robertianum L.

Hedera helix L.

Urginea maritima (L.) Baker

Acanthus mollis L.

Sporadiche $\begin{array}{lllllllllllllllll}1 & 2 & 3 & 4 & 5 & 6 & 7 & 8 & 9 & 10 & 11 & 12 & 13 & 14 & 15 & 16 & 17\end{array}$

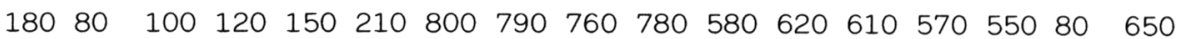
$\begin{array}{lllllllllllllllll}50 & 50 & 50 & 40 & 50 & 50 & 50 & 100 & 50 & 100 & 80 & 50 & 100 & 200 & 200 & 100 & 100\end{array}$ $10010010010010010090 \quad 100 \quad 100 \quad 100 \quad 100 \quad 100 \quad 10095 \quad 90 \quad 100 \quad 100$

$\begin{array}{llllllllllllllllll}5 & 5 & 5 & 4 & 5 & 5 & 4 & 3 & 4 & 4 & 4 & 5 & 5 & 4 & 4 & 5 & 5 \\ 1 & 2 & 2 & \cdot & \cdot & 2 & 2 & 2 & 2 & 2 & 3 & 2 & + & 2 & 3 & 1 & 1 \\ 2 & 2 & 3 & 3 & 3 & 2 & 1 & 2 & 1 & 2 & 1 & 1 & 2 & + & 1 & 1 & + \\ \cdot & \cdot & \cdot & \cdot & \cdot & \cdot & \cdot & 2 & + & \cdot & \cdot & + & + & + & \cdot & + & \cdot \\ + & 1 & + & + & \cdot & + & 1 & 1 & \cdot & \cdot & + & 1 & . & \cdot & + & \cdot & \cdot \\ \cdot & \cdot & + & \cdot & 1 & \cdot & \cdot & \cdot & + & \cdot & + & 1 & . & . & . & . & .\end{array}$

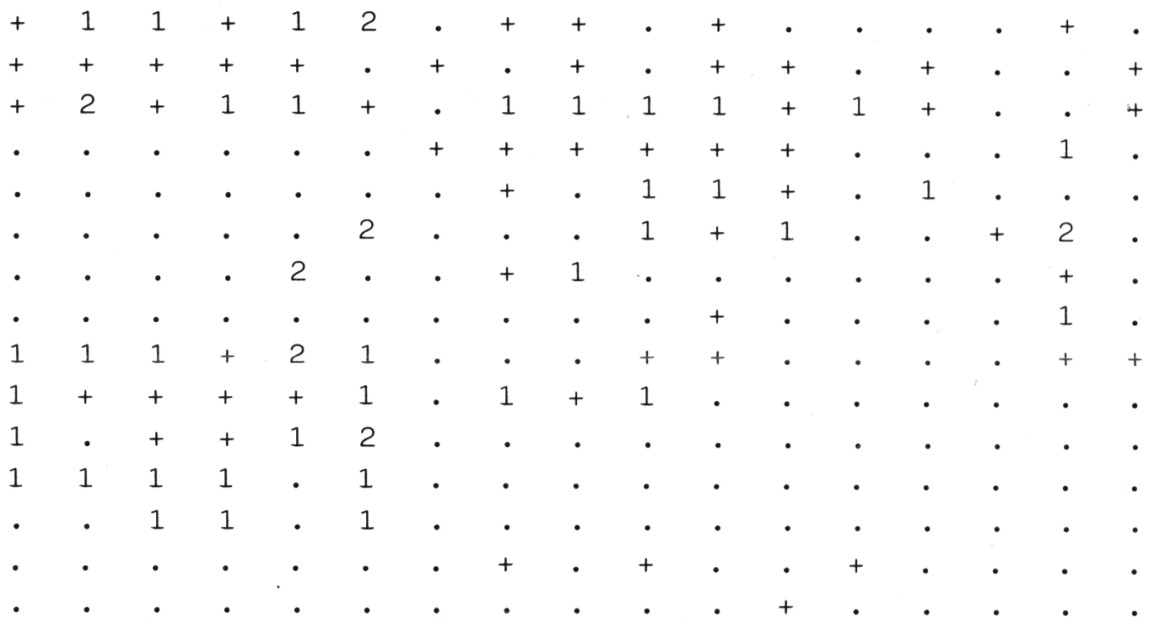




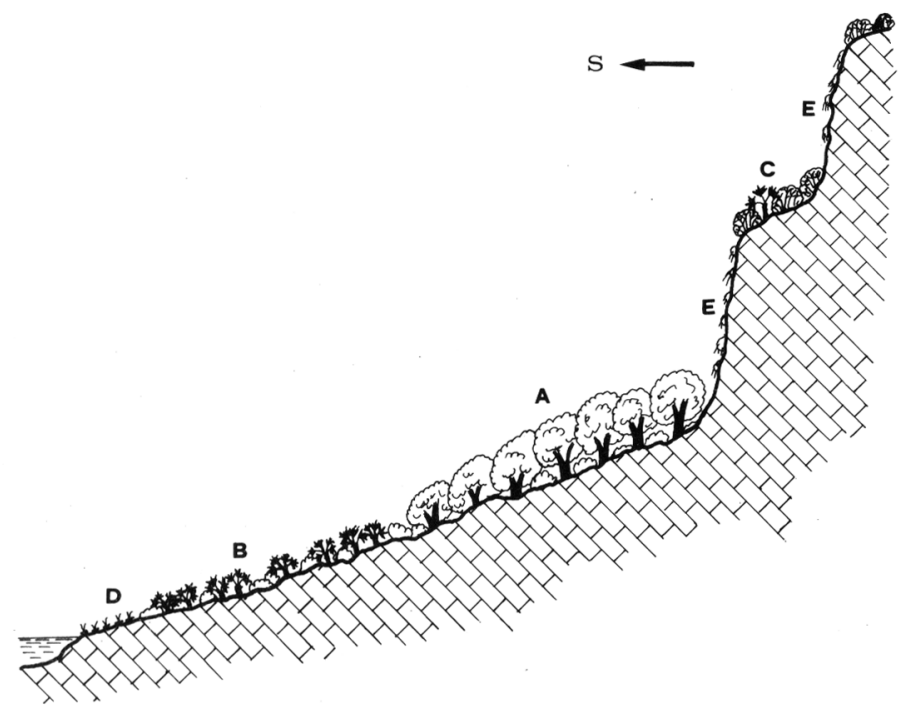

Figura 5.- Transect della vegetazione potenziale della fascia costiera della Sicilia nord-occidentale: A. Rhamno alatherni-Quercetum ilicis; B. Pistacio-Chamaeropetum humilis; C. Oleo-Euphorbietum dendroidis; D. Limonietum bocconei; E. Scabioso-Centauretum ucriae.

E - Aceri campestris-Quercetum ilicis Brullo 1984 (Tab. 5, Fig. 6)

Si tratta di una lecceta orofila distribuita sui rilievi montuosi calcarei e dolomitici della Sicilia nord-occidentale. In particolare essa è stata rilevata sulle Madonie e a Rocca Busambra a quote comprese fra 1000 e $1500 \mathrm{~m}$, dove si insedia su brecciai consolidati e in stazioni semirupestri. Sotto il profilo ecologico questa lecceta risulta legata ad un suolo poco evoluto, in genere piuttosto superficiale, e ad un clima di tipo mediterraneo-montano caratterizzato da precipitazioni medie annue superiori a $1000 \mathrm{~mm}$.

Differenziali dell'associazione sono alcune orofite arboree come Ilex aquifolium, Acer campestris, Acer monspessulanus e Sorbus graeca.

L'Aceri campestris-Quercetum ilicis, a differenza delle altre leccete già esaminate, assume un ruolo prettamente climacico costituendo l'aspetto vegetazionale più evoluto del piano submontano. Esso infatti al di sotto dei $1000 \mathrm{~m}$ sfuma verso l'Oleo-Quercetum virgiliane Brullo 1984, mentre in alto, al di sopra dei 14001500 m prende contatto con l'Anemono-Fagetum (Gentile 1969) Brullo 1984, faggeta termofila che ricopre i versanti dei rilievi fino a $1800-1900 \mathrm{~m}$ di quota.

In ambienti prettamente rupestri esso viene sostituito da una tipica vegetazione casmofila rappresentata dall'Anthemido-Centauretum busambarensis Brullo \& Marcenò 1979, associazione degli Asplenietalia glandulosi.

Dal punto di vista sindinamico questo tipo di lecceta risulta collegata con le associazioni arbustivo-pulvinari del Seslerio-Astragalion nebrodensis Pignatti \& Nimis ex Brullo 1984, le quali tendono a sostituirla in seguito ai processi di degradazione che portano alla distruzione dello strato arboreo. 


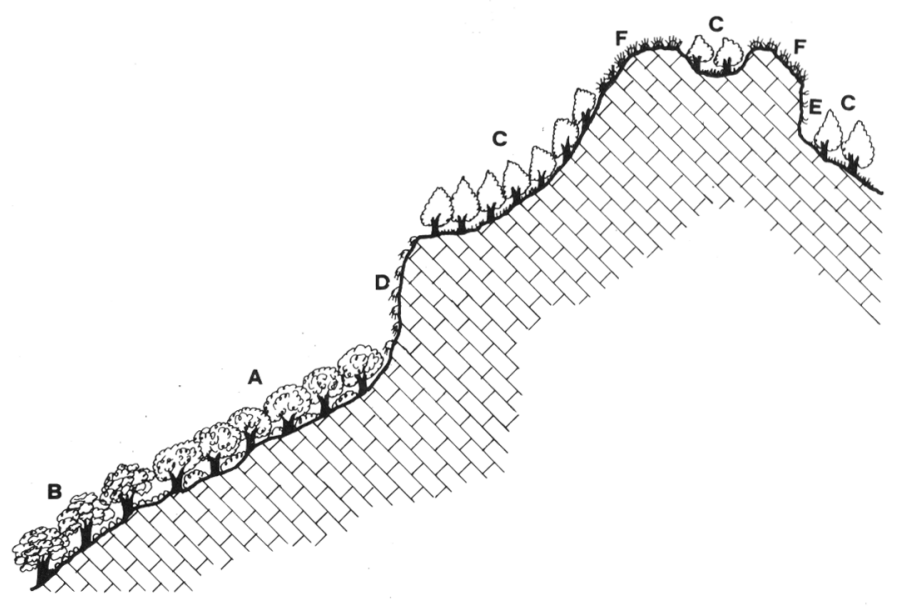

Figura 6.- Transect della vegetazione potenziale calcicola delle Madonie (Sicilia settentrionale):

A. Aceri campestris-Quercetum ilicis; B. Oleo-Quercetum virgilianae; C. AnemonoFagetum; D. Anthemido-Centauretum busambarensis; E. Asperuletum gussonei; F. Seslerio-Astragalion nebrodensis.

F-Erico-Quercetum ilicis Brullo, Di Martino \& Marcenò 1977 (Tab. 6, Fig. 7)

Questa associazione è stata osservata soprattutto in stazioni costiere di tipo insulare, aree queste interessate da un clima prettamente marittimo influenzato durante buona parte dell'anno da precipitazioni occulte.

Si tratta di una associazione calcifuga rilevata in alcune isole circumsiciliane di natura vulcanica, quali Pantelleria e l'Arcipelago delle Eolie; mentre rari esempi si rinvengono all'interno della Sicilia sulle vulcaniti iblee in prossimità di Mineo. Floristicamente essa risulta differenziata da sclerofille dei Pistacio-Rhamnetalia alaterni, fra cui soprattutto Pistacia lentiscus, Myrtus communis, Prasium majus, ecc., specie queste che confermano una certa xerofihia dell'associazione. In effetti, l'Erico-Quercetum ilicis è da considerare come la più termofila fra le leccete acidofile dell'Erico-Quercion ilicis. Per quanto riguarda la sua distribuzione generale, essa risulta diffusa, sulla base dei dati di letteratura (cfr. Brullo \& Marcenò 1985), sia nei territori circumtirrenici (Francia meridionale, Peninsola italiana, Corsica, Sardegna e Sicilia) che nella Peninsola balcanica dove si insedia su substrati di varia natura, ma soprattutto su vulcaniti, graniti, scisti e gneiss; si rinviene talora anche su calcare ma limitatamente a territori interessati da precipitazioni abbastanza elevate, in genere superiori a $800 \mathrm{~mm}$ annui, che favoriscono i processi di lisciviazione del suolo.

Per quanto riguarda le stazioni sicule, l'Erico-Quercetum ilicis rappresenta, limitatamente ai territori in cui è stato rilevato, la vegetazione forestale più evoluta, assumendo pertanto un carattere climacico. In particolare a Pantelleria questa lecceta si insedia nelle zone cacuminali, stazioni queste interessate per buona parte dell'anno da un regime di nebbie. Verso il basso, al disotto dei 600-700 m, essa prende contatto con il Pino-Genistetum aspalathoidis Brullo, Di Martino \& Marcenò 1977, pi- 
Numero del rilievo

Quota (m)

Superficie (mq)

Copertura (\%)

Inclinazione $\left({ }^{\circ}\right)$

Esposizione

Diff. Associazione

Teucrium siculum Rafin.

Diff. Subass. (POLYSTICHETOSUM)

Polystichum setiferum (Forsskal) Woynar

Car. Suball. (QUERCENION DELECHAMPII)

e All. (ERICO-QUERCION ILICIS)

Erica arborea L.

Quercus dalechampii Ten.

Quercus congesta Presl

Festuca exaltata C.Presl

Clinopodium vulgare L.ssp.arundarum (Boiss.) Nyman Arbutus unedo $\mathrm{L}$.

Cytisus villosus Pouret

Melica arrecta Kuntze

Pulicaria odora (L.) Rchb.

Teline monspessulana (L.) Koch

Car. Ord. (QUERCETALIA ILICIS)

e $\mathrm{Cl}$. (QUERCETEA ILICIS)

Quercus ilex L.

Buscus aculeatus L.

Asplenium onopteris L.

Cyclamen hederifolium Aiton

Rubia peregrina L.

Carex distachya Desf.

Viola dehnhardtii Ten.

Euphorbia characias L.

Asparagus acutifolius L.

Fraxinus ornus L.

Calicotome infesta (C.Presl) Guss.

Daphne gnidium L.

Rosa sempervirens $\mathrm{L}$.

Ostrya carpinifolia Scop.

Smilax aspera L.

Phillyrea latifolia

Luzula forsteri (Sm.) DC.

Cyclamen repandum Sibth. \& Sm.

Lonicera etrusca Santi

Tamus communis L.

Arisarum vulgare Targ.-Tozz.

Pimpinella peregrina $L$.

Pirus amygdalifformis Vill.

Osyris alba L.

Carex hallerana Asso

Lonicera implexa Aiton

Phillyrea angostifolia L.

Altre specie

Brachypodium sylvaticum (Hudson) Beauv.

Pteridium aquilinum (L.) Kuhn

Hedera helix L.

Rubus ulmifolius Schott

Malus sylvestris Miller

Crepis leontodontoides All.

Agrimonia eupatoria L.

Asplenium trichomanes $\mathrm{L}$.

Clematis vitalba $\mathrm{L}$.

Silene italica (L.) Pers.

Quercus cerris

Calamintha grandiflora (L.) Moench

Prunus spinosa L.

Festuca heterophylla Lam.

Anthoxanthum odoratum L.

Dactylis hispanica Roth

Selaginella denticulata (L.) Link

Ranunculus neapolitanus Ten.

Geranium purpureum Vill.

Rosa canina L.

Allium subhirsutum L.

Crataegus monogyna Jacq.

Achillea ligustica All.

Micromeria graeca (L.) Bentham

Hypericum perforatum L.

Sporadiche $\begin{array}{lllllllllllllllllll}1 & 2 & 3 & 4 & 5 & 6 & 7 & 8 & 9 & 10 & 11 & 12 & 13 & 14 & 15 & 16 & 17 & 18 & 19\end{array}$

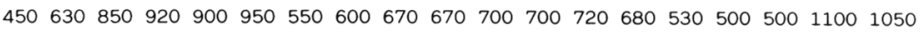
$\begin{array}{lllllllllllllllllll}100 & 100 & 100 & 100 & 100 & 100 & 100 & 100 & 100 & 100 & 100 & 100 & 50 & 100 & 50 & 60 & 50 & 100 & 60\end{array}$ $\begin{array}{lllllllllllllllllll}100 & 100 & 100 & 100 & 100 & 100 & 100 & 100 & 100 & 100 & 100 & 100 & 100 & 100 & 100 & 100 & 100 & 100 & 100\end{array}$ $\begin{array}{lllllllllllllllllll}20 & 25 & 20 & 15 & 20 & 30 & 20 & 20 & 30 & 25 & 25 & 40 & 50 & 45 & 45 & 40 & 30 & 25 & 30 \\ \mathbf{N} & \mathrm{N} & \mathrm{N} & \mathrm{N} & \mathrm{N} & \mathrm{N} & \mathrm{N} & \mathrm{N} & \mathrm{N} & \mathrm{N} & \mathrm{N} & \mathrm{N} & \mathrm{N} & \mathrm{N} & \mathrm{N} & \mathrm{N} & \mathrm{N} & \mathrm{N} & \mathrm{N}\end{array}$

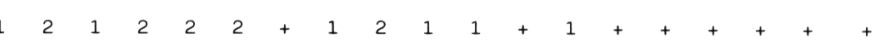

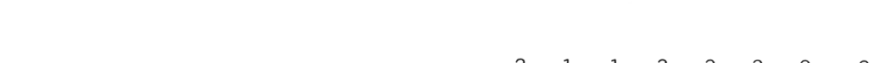


neta termofila ricoprente buona parte dei versanti dei rilievi dell'isola (cfr. Brullo et al. 1977). Anche nelle isole Eolie la lecceta tende a ricoprire le parti più alte dei coni vulcanici sostituendosi al Genistetum tyrrheniae Brullo nom. nov. (= Genistetum ephedroidis Brullo, Di Martino \& Marcenò 1977 nom. inval.), vegetazione arbustiva termofila del Cisto-Ericion Horvatic 1958. Esempi di leccete si rinvengono pure nelle parti basse di queste isole in corrispondenza però di valloni o di zone di compluvio, superfici queste caratterizzate da una umidità edafica più marcata rispetto alle superfici circostanti.

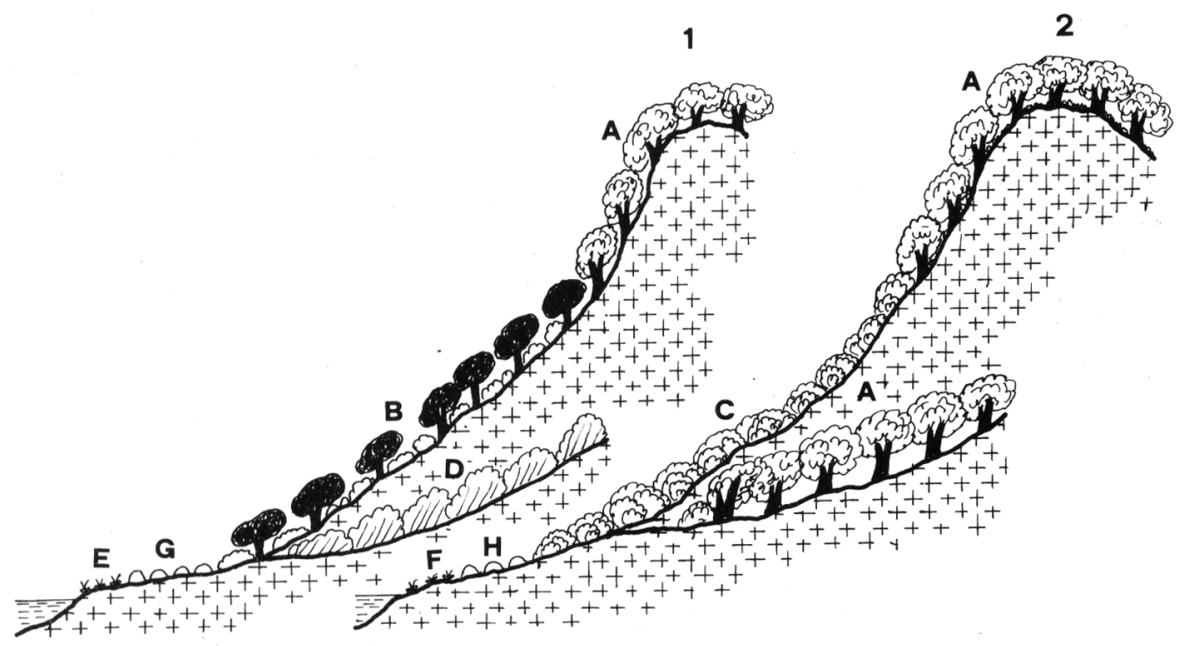

Figura 7.- Transect della vegetazione potenziale di Pantelleria (1) e delle Isole Eolie (2): A. Erico-Quercetum ilicis; B. Pino pinastris-Genistetum aspalathoidis; C. Genistetum tyrrheniae; D. Erico arboreae-Juniperetum phoeniciae; E. Limonietum cossyrensis; F. Limonietum minutiflori; G. Matthiolo-Helichrysetum errerae; H. Senecioni-Helichrysetum litorei.

G-Querco-Teucrietum siculi Gentile 1969 em. Brullo \& Marcenò 1985 (Tab.7, Fig. 8)

Sui rilievi della Sicilia settentrionale e nord-orientale, limitatamente ai substrati silicei (schisti, gneiss, graniti, flisch) è presente una lecceta acidofila nettamente più mesofila dell'associazione precedente, la quale mostra in genere un significato azonale. Questa vegetazione, rappresentata dal Querco-Teucrietum siculi, associazione descritta da Gentile (1969) e successivamente emendata da Brullo \& Marcenò (1985), si localizza infatti sul fondo di valloni e sui versanti più ombreggiati dei rilievi. Si tratta pertanto di un aspetto forestale legato a condizioni ambientali particolarmente umide e fresche di tipo microclimatico.

Specie guida dell'associazione è Teucrium siculum, entità endemica della Sicilia e dell'Appennino meridionale, la quale permette di differenziare abbastanza bene quest'aspetto dalle altre leccete note in letteratura. Fra queste il Querco-Teucrietum siculi mostra una maggiore affinità con l'Erico-Quercetum ilicis, ma da questo 
si distingue abbastanza bene, oltre che per l'ecologia, anche per la presenza di alcune specie caratteristiche della suball. Quercenion dalechampii Brullo 1984, quali Quercus dalechampii, Quercus congesta, Festuca exaltata, Clinopodium vulgare ssp. arundanum, specie queste prettamente mesofile. zioni:

In seno al Querco-Teucrietum siculi possono essere distinte due subassocia-

1 -teucrietosum siculi subass. nov. (olotipo: lo stesso dell'associazione) - Essa rappresenta l'aspetto tipico dell'associazione e risulta differenziata dalla maggiore abbondanza di Teucrium siculum, nonchè dalla presenza delle specie più termofile dell'Erico-Quercion ilicis, come Arbutus unedo, Cytisus villosus, Melica arrecta, Pulicaria odora, Teline monspessulana.

2 -polystichetosum setiferi subass. nov. (olotipo: ril. 15, Tab. 7) Questo aspetto si localizza nelle stazioni più umide come il fondo di vallecole e le aree di compluvio ed ha come specie differenziale Polystichum setiferum.

Riguardo alla sua distribuzione, esso è presente sia in Sicilia che nell'Appennino meridionale (cfr. Gentile 1969, Barbagallo et al. 1982, Brullo \& Marcenò 1985, Signorello 1986, Schneider \& Sutter 1982). Si rinviene dal piano basale fino a quello montano, a quote comprese tra 400 e $1300 \mathrm{~m}$ in territori interessati da precipitazioni medie annue di 900-1200 mm.

In Sicilia questa lecceta è diffusa all'interno della fascia climacica di querceti caducifogli, fra cui in particolare il Quercetum leptobalanae Brullo 1984 per quanto riguarda le Madonie e l'Erico-Quercetum virgilianae Brullo \& Marcenò 1985 nel caso dei Nebrodi e dei Peloritani.

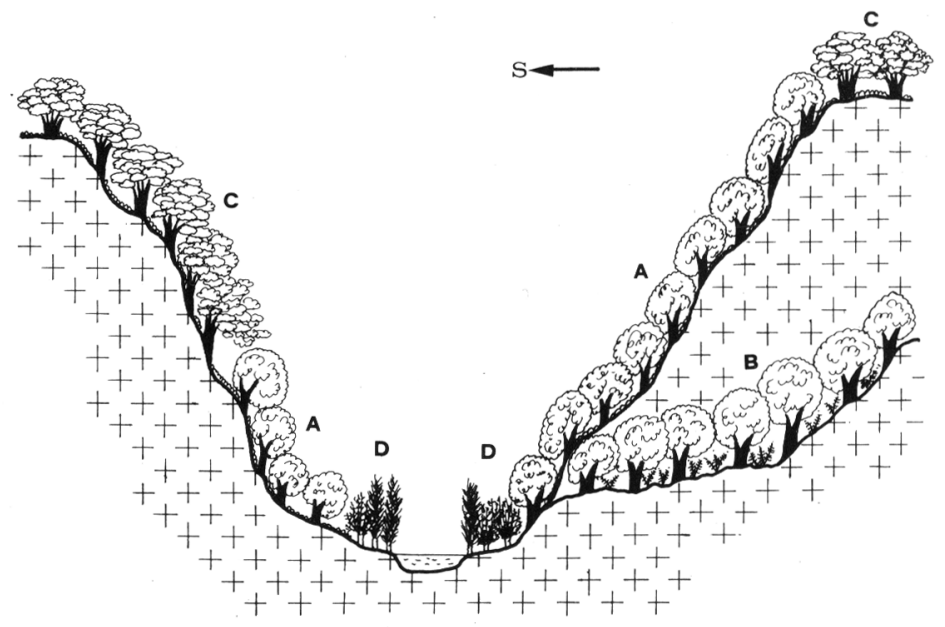

Figura 8.- Transect della vegetazione potenziale calcifuga delle Madonie (Sicilia settentrionale):

A. Querco-Tencrietum siculi teucrietosum siculi; B. Querco-Teucrietum siculi polystichetosum setiferi; C. Quercetum leptobalanae; D. Ulmo canescentis-Salicetum pedicellatae. 
Numero del rilievo

Quota (m)

Superficie (mq)

Copertura (\%)

Inclinazione $\left({ }^{\circ}\right)$

Car. Associazione

Ostrya carpinifolia Scott

Acer obtusatum W. \& $K$.

Diff. Subass. (TILIETOSUM)

Tilia platyphyllos Scop.

Laurus nobilis L.

Car. Suball. (QUERCENION DALESCHAMPII)

e AIl. (ERICO-QUERCION ILICIS)

Quercus daleschampii Ten.

Cytisus villosus Pourret

Quercus congesta C.Presl

Festuca exaltata C.Presl

Teucrium siculum Rafin.

Poa sylvicola Guss.

Silene viridifolia L.

Clinopodium vulgare L.ssp. arundarum (Boiss.) Nyman Erica arborea L.

Melica arrecta Kuntze

Pulicaria odora (L.) Reichenb.

Car. Ord. (QUERCETALIA ILICIS)

e Cl. (QUERCETEA ILICIS)

Quercus ilex L.

Fraxinus ornus L.

Asplenium onopteris L.

Carex distachya Desf.

Ruscus aculeatus L.

Asparagus acutifolius L.

Rubia peregrina L.

Lonicera etrusca Santi

Euphorbia characias L.

Cyclamen hederifolium Aiton

Quercus amplifolia Guss.

Thalictrum calabricum Sprengel

Rhamnus alaternus L.

Tamus communis $\mathrm{L}$.

Smilax aspera $\mathrm{L}$.

Luzula forsteri (Sm.) DC.

Bupleurum fruticosum $\mathrm{L}$.

Viola dehnhardtii Ten.

Polystichum setiferum (Forsskal) Woynar

Teucrium flavum L.

Pirus amygdaliformis Vill.

Rosa sempervirens L.

Cyclamen repandum Sibth. \& Sm.

\section{Altre specie}

Pteridium aquilinum (L.) Kuhn

Brachypodium sylvaticum (Hudson) Beauv.

Crepis leontodontoides All.

Rubus aetnicus Ten.

Crataegus monogyna Jacq.

Carlina corymbosa $L$.

Achillea ligustica All.

Trifolium ochroleucum Hudson

Hypericum perforatum L.

Pinus laricio Poiret

Asplenium trichomanes L.

Bellis perennis L.

Lolium rigidum Gaudin

Origanum vulgare $\mathrm{L}$.

Spartium junceum L.

Daucus carota L.

Castanea sativa Miller

Rosa canina L.

Polypodium cambricum Willd.

Silene vulgaris (Moench) Garcke

Geranium purpureum Vill.

Acer pseudoplatanus L.

Hedera helix L.

Rubus canescens DC.

Clematis vitalba $\mathrm{L}$.

Saxifraga rotundifolia $\mathrm{L}$.

Geranium versicolor L.

Sporadiche

Ril. 1-6 Etna (BRULLO \& MARCENO' 1985)

Ril. 7-12 Vallone Santissima, Peloritani $\begin{array}{llllllllllll}1 & 2 & 3 & 4 & 5 & 6 & 7 & 8 & 9 & 10 & 11 & 12\end{array}$ $\begin{array}{llllllllllll}620 & 750 & 680 & 800 & 720 & 750 & 780 & 670 & 670 & 670 & 670 & 670\end{array}$

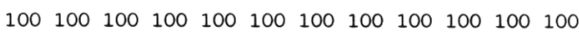

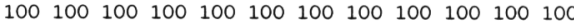
$\begin{array}{llllllllllll}20 & 25 & 20 & 20 & 20 & 25 & 60 & 70 & 80 & 70 & 70 & 60\end{array}$
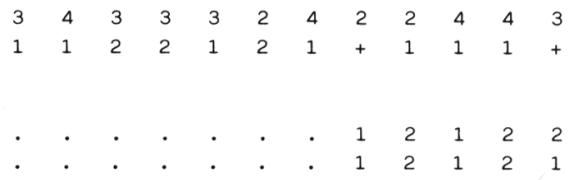
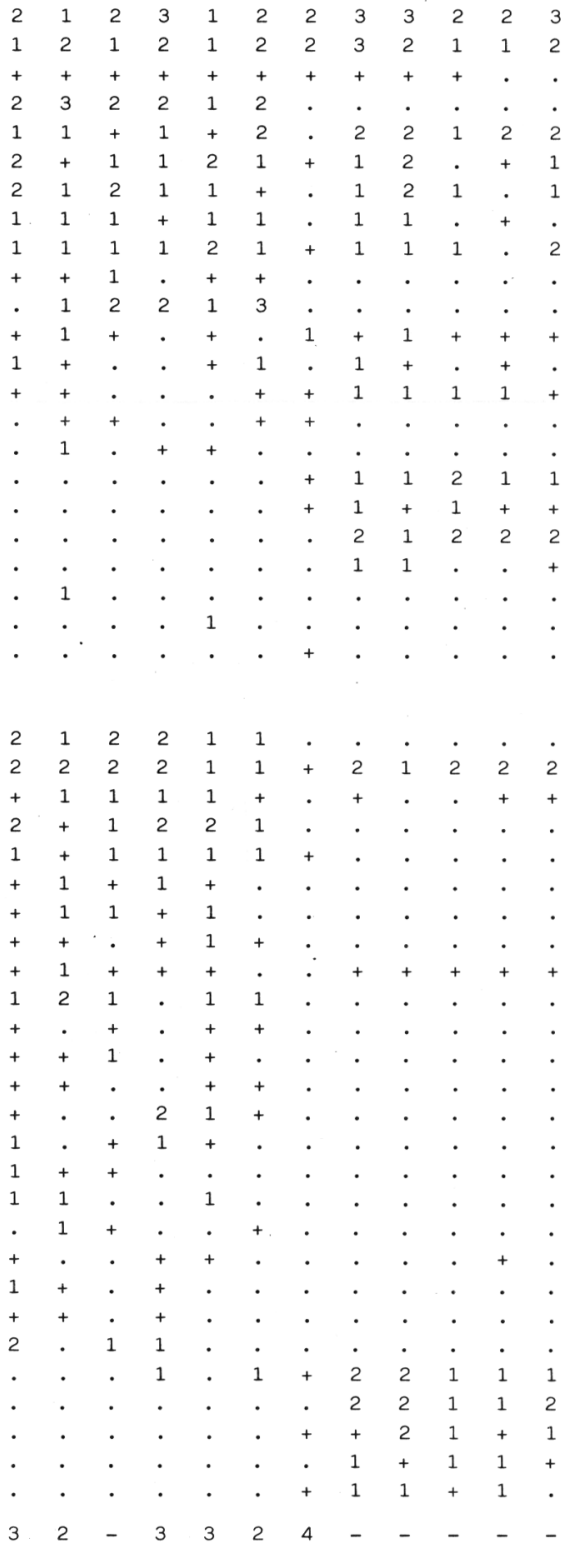
H - Aceri obtusati-Ostryetum carpinifoliae Brullo \& Marcenò 1985 (Tab. 8, Fig. 9)

Questa associazione rappresenta un tipo di vegetazione boschiva molto peculiare nella quale Quercus ilex non assume un ruolo dominante, ma questa sclerofilla sempreverde si accompagna a diverse essenze legnose caducifoglie di tipo mesofilo, come Ostrya carpinifolia, Acer obtusatum, Fraxinus ornus, Quercus dalechampii e Quercus congesta. Essa appare legata a substrati silicei di stazioni submontane caratterizzate da condizioni ambientali molto più fresche ed umide rispetto al territorio circostante. Si tratta comunque di un aspetto abbastanza raro in Sicilia, inizialmente rinvenuto solo sul versante orientale dell'Etna (cfr. Brullo \& Marcenò 1985). Ulteriori ricerche hanno permesso di accertarne la sua presenza anche sul versante orientale dei Peloritani. Nel complesso questa associazione risulta distribuita in aree interessate da un clima di tipo umido-subumido con precipitazioni medie annue superiori a $900-1000 \mathrm{~mm}$.

Nell'ambito dell'Aceri obtusati-Ostryetum carpinifoliae si possono distinguere le seguenti subassociazioni:

1 -quercetosum congestae subass. nov. (olotipo: stesso dell'associazione) - Rappresenta l'aspetto tipico dell'associazione ed è circoscritto alle vulcaniti etnee. Specie differenziale è Quercus congesta.

2 -tilietosum platyphylli subass. nov. (olotipo: ril. 11, Tab. 8) Questo aspetto è stato rilevato sui Peloritani presso Fiumedinisi in ambienti di forra profonda molto umida e ombreggiata. Esso ricopre i versanti fortemente inclinati di questi peculiari habitat rupestri costituendo una vegetazione molto densa. Rilevante è la presenza di Tilia platyphyllos, specie in Sicilia rarissima fin ora nota solo per questa località dei Peloritani, e di Laurus nobilis. Queste specie, che vengono proposte come caratteristiche della subassociazione, sono da considerare come degli elementi relitti, aventi in questi habitat le loro stazioni di rifugio.

3 -ostryetosum carpinifoliae subass. nov. (olotipo: ril. 8 Tab. 8) Nella parte alta più aperta della forra interessata dalla subassociazione precedente si insedia una vegetazione ascrivibile sempre all'Aceri obtusati-Ostryetum carpinifoliae, ma caratterizzata dalla dominanza di Ostrya carpinifolia. Essa è da considerare come un aspetto legato sempre a condizioni microclimatiche abbastanza fresche ed umide, ma con esigenze più eliofile. Questa vegetazione nella stazione più aperte e soleggiate viene infatti sostituita del tutto dall'Erico-Quercetum virgilianae Brullo \& Marcenò 1985 associazione boschiva più termofila rappresentante la vegetazione climacica di questa parte dei Peloritani. 


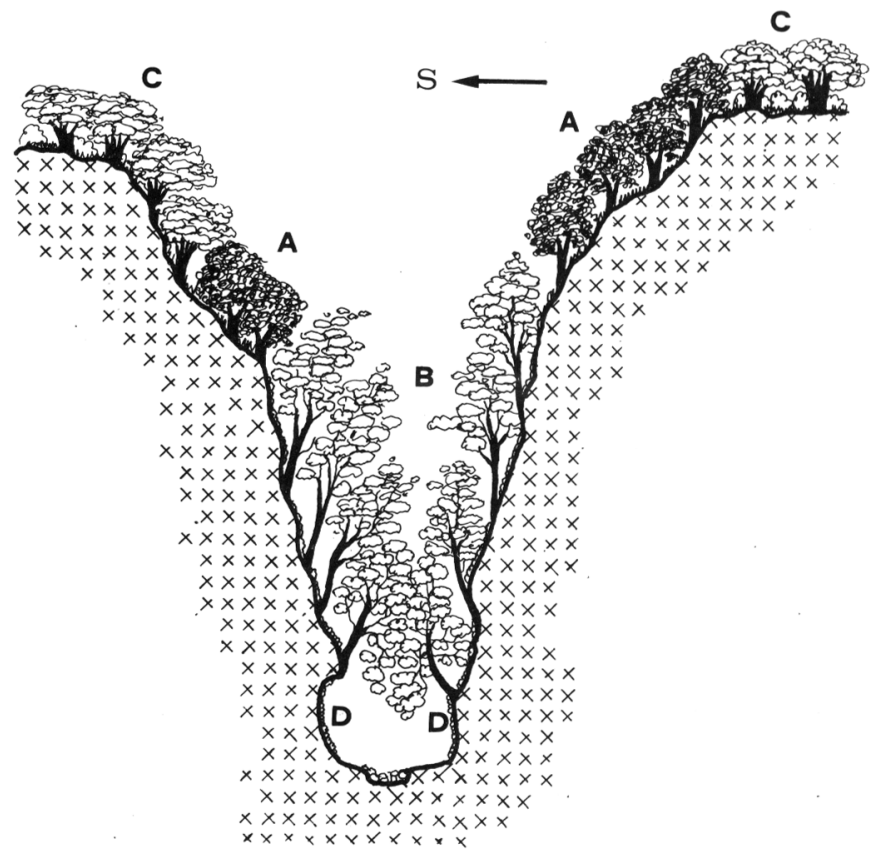

Figura 9.- Transect della vegetazione di forra dei Peloritani (Sicilia nord-orientale): A. Aceri obtusati-Ostryetum carpinifoliae ostryetosum; B. Aceri obtusati-Ostryetosum carpinifoliae tilietosum platyphylli; C. Erico-Quercetum virgilianae; D. Thamnobryetum alopecuri.

R I N G R A Z I A M E N T I . Lavoro eseguito con il supporto finanziario del Consiglio Nazionale delle Ricerche (C.N.R. Italia), che qui si ringrazia.

\section{B I B L I O G R A F I A}

BALLELLI, S. \& BIONDI, E. -1982- Carta della vegetazione del foglio Pergola. CNR AQ/1/130 Roma.

BARBAGALLO, C., BRULLO, S. \& FAGOTTO, F. -1979- Boschi di Quercus ilex del territorio di Siracusa e principali aspetti di degradazione. Publ. Ist. Bot. Univ. Catania.

BARBAGALLO, C., BRULLO, S. \& FAGOTTO, F. -1979a- Vegetazione a Platanus orientalis L. ed altri aspetti igrofili dei fiumi iblei (Sicilia meridionale). Publ. Ist. Bot. Univ. Catania.

BARBAGALLO, C., BRULLO, S., FURNARI, F., LONGHITANO, N. \& SIGNORELLO, P. - 1982- Studio fitosociologico e cartografia della vegetazione (1:25000) del territorio di Serra S. Bruno (Calabria). CNR AQ/1/227 Roma.

BIONDI, E. -1982- L'Ostrya carpinifolia Scop. sul litorale delle Marche (Italia centrale). Studia geobotania, 2:141-147.

BLASI, C., ABBATE, G., FASCETTI, S. \& MICHETTI, L. -1981- La vegetazione del bacino del F. Treia (complesso vulcanico Sabatino e Vicano, Lazio nord-occidentale). CNR AQ/1\&237. Roma. 
BRULLO, S. -1984- Contributo alla conoscenza della vegetazione delle Madonie (Sicilia settentrionale). Boll. Acc. Gioenia Sci. Nat., 16(322):351-420.

BRULlo, S., DI MARTINO, A. \& MARCENO, C. -1977- La vegetazione di Pantelleria (Studio fitosociologico). Pubbl. Ist. Univ. Catania.

BRULLO, S. \& MARCENO, C. -1979- Dianthion rupicolae, nouvelle alliance sud-tyrrhenienne des Asplenietalia glandulosi. Doc. Phytosoc., n.s., 4:131-146.

BRULLO, S. \& MARCENO, C. -1985- Contributo alla conoscenza della classe Quercetea ilicis in Sicilia.Not. Fitosoc., 19(1):183-229.

BRULLO, S. \& RONSISVALLE, G.A. - 1975- La vegetazione dei Gorghi Tondi e del Lago Preola preso Mazara del Vallo (Sicilia occidentale). Not. Fitosoc., 10:45-67.

FURNARI, F. -1965- Boschi a Quercus suber L. e di Quercus ilex L. e le garighe del Rosmarino-Ericion in territorio di Santo Pietro (Sicilia meridionale). Boll. Ist. Bot. Univ. Catania, s. 3, 5:1-31.

GENTILE, S. -1969- Remarques sur les chènaies d'Yeuse de l'Apenin méridional et de la Sicile. Vegetatio, 17:214-231.

LAPRAZ, G. -1975- Les groupements végétaux de la classe Quercetea ilicis dans les Alpes Maritimes orientales calcaire entre Le Var et Menton (Synthese phytosociologique). Anal. Inst. Bot. Cavanilles, 32:1183-1208.

POLI, E. \& MAUGERI, G. -1974- I boschi di Leccio del versante nord-occidentale dell'Etna. Boll. Acc. Gioenia Sci. Nat. Catania, s. 4, 12(5-6):741-759.

POLI, E., LO GIODICE, R. \& FERLITO, N. -1979- La vegetazione della valle San Giacomo (Etna). Atti, Acc. Gioenia Sci. Nat. Catania, s. 7, 10:253-319.

SIGNORELLO, P. -1985- Osservazioni fitosociologiche su alcuni aspetti boschivi dei Quercetea ilicis dell'Italia meridionale. Not. Fitosoc., 19(1):177-181.

SIGNORELLO, P. -1986- Osservazioni fitosociologiche sulla vegetazione dell'Aspromonte (Calabria meridionale). Pubbl. Ist. Bot. Univ. Catania.

SCHNEIDER, R. \& SUTTER, R. -1982- Beitrag zur Flora und Vegetation Südkalabriens. Phytocoenologia. 10(3):323-373.

TRINAJSTIC, I. -1977- Osnovne znacajke biljnog pokrova otoka Hvara injegov fitogeograsvki polozas u okviru Evropskog dijela Sredozemlja. Poljoprivreda I Sumartvo, 23(4):1-36.

(Aceptado para sú publicación el 12 de abril de 1990)

Dirección de los autores: G. BARTOLO, S. BRULLO \& P. MINISSALE: Istituto ed Orto Botanico, Universitá di Catania, via A. Longo 19, Catania, I-95125. G. SPAMPINATO: Istituto Chimica agraria e forestale. Facoltà di Agraria, Università di Reggio Calabria, piazza S. Francesco, Gallina, Reggio Calabria. 